\title{
Specimen Inertia in high strain rate tensile testing
}

\author{
Meyrick Hockly and Clive R. Siviour ${ }^{\mathrm{a}}$ \\ Department of Engineering Science, University of Oxford, Parks Road, Oxford OX1 3PJ, UK
}

\begin{abstract}
It is well established that measurements of the dynamic mechanical response of materials are susceptible to errors due to the inertia of the specimen, which causes stresses in addition to the intrinsic material strength. A number of authors have derived equations for these stresses in compression experiments; these equations can be used as a guideline for good specimen design. However, no such equations have been presented in the literature for the equivalent effects in tensile experiments. This paper begins by considering and rationalising the equations available for compression, before producing a set of equations which can be used in design of specimens for, e.g., tensile Hopkinson bar experiments.
\end{abstract}

\section{Introduction}

It has long been known in the high rate testing community $[1,2]$ that the apparent strength of any test specimen is a combination of the intrinsic material strength, and any effects due to specimen inertia. For loading at low rates of strain, the effect of inertia is minimal; however, it can be shown [1-5] that for large specimen sizes, dense materials, or materials with low intrinsic strength, the additional stresses required to overcome the axial and radial inertia of the specimen can represent a significant fraction of the measured loads in a high rate characterisation experiment. Indeed, as the inertial loads scale with the square of strain rate $\left(\dot{\varepsilon}^{2}\right)$ and rate of change of strain rate $\left(\ddot{\varepsilon}^{2}\right)$, their effects can rapidly become significant in dynamic testing.

A number of authors have derived equations to quantify the effects of specimen inertia in compressive Hopkinson bar experiments. The calculations can be divided into two categories: those which use an energy based approach, and those which use calculations of the stress-field in the specimen. Both approaches require some approximations to the behaviour of a real specimen.

This paper will begin by examining the different equations derived for compressive inertia, reconciling the different expressions obtained by the energy and stress based approaches. Then, extending the energy based analysis, expressions will be derived for the effects of inertia in a tensile experiment. Firstly, tension will simply be considered as a reverse compression on a long cylinder. Then, point masses will be added to mimic the ends of a dog-bone tensile specimen, before moving to an analysis which considers a more realistic specimen geometry.

\section{Inertia in compression specimens}

\subsection{Definition of variables}

This paper will adopt a similar nomenclature to Gorham, which in the first instance is designed to simplify the

\footnotetext{
${ }^{a}$ Corresponding author: clive.siviour@eng.ox.ac.uk
}

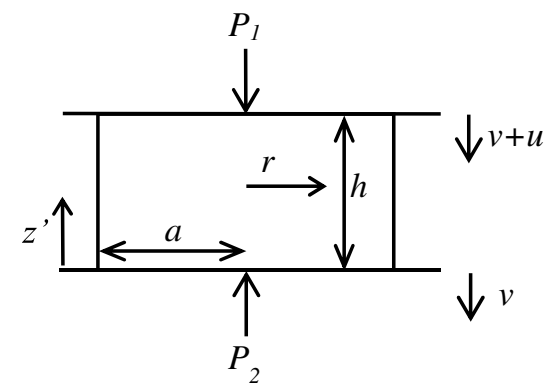

Figure 1. Definition of symbols used in this paper. $P_{1}$ and $P_{2}$ are the mean pressures between the specimen and input and output bars respectively. The output bar moves at speed $v$. The $\left(\theta, r, z^{\prime}\right)$ coordinate system is shown and is defined in the moving frame such that $z^{\prime}=0$ (always) at the bottom of the specimen and $h$ (instantaneously) at the top; $r=0$ at the axis and $a$ at the outer edge. There is also an inertial $\left(\theta, r, z^{\prime}\right)$ system in which the bottom of the specimen has axial velocity $-v$, the rest of the specimen $-v-u_{z}(z)=-v-u z / h$. The radial velocity is $u_{r}(r)$.

equations in compression. Later some of the definitions will be altered for the tension work. The symbols are defined as in Fig. 1. Strain is positive in tension.

\subsection{Energy based approaches}

A number of authors have considered the effects of specimen inertia by comparing the work done by the forces applied to the two ends of the specimen by the Hopkinson bars to the combination of kinetic and deformation energy in the specimen. The first approaches by Davies and Hunter [2] and Samanta [3] made a number of assumptions which ignored potentially significant terms: in particular assuming that the output end of the specimen was stationary. Gorham [4] considered a more general case, but still assuming that the strain and stress fields in the specimen are everywhere the same at any time. He derived the following equations for the stresses at the interface

This is an Open Access article distributed under the terms of the Creative Commons Attribution License 4.0, which permits unrestricted use, distribution, and reproduction in any medium, provided the original work is properly cited. 
between the input bar and the specimen $\left(P_{1}\right)$ and the interface between the specimen and the output bar $\left(P_{2}\right)^{1}$

$$
\begin{aligned}
& P_{1}=\sigma_{y}-\rho \ddot{\varepsilon}\left(\frac{a^{2}}{8}+\frac{h^{2}}{3}\right)+\rho \dot{\varepsilon}^{2}\left(\frac{a^{2}}{16}-\frac{h^{2}}{3}\right)+\frac{\rho h \dot{v}}{2} ; \\
& P_{2}=\sigma_{y}-\rho \ddot{\varepsilon}\left(\frac{a^{2}}{8}-\frac{h^{2}}{6}\right)+\rho \dot{\varepsilon}^{2}\left(\frac{a^{2}}{16}-\frac{h^{2}}{6}\right)+\frac{\rho h \dot{v}}{2} ;
\end{aligned}
$$

where $a$ and $h$ are the specimen radius and height, $\rho$ the density, $\varepsilon$ the strain rate and $v$ the velocity of the interface between the specimen and the output bar. It is noted that in this case, the volume of the specimen is assumed to be constant during the experiment and that the strain and strain rate are negative in compression. Further, following Gorham, $\sigma_{y}$ is the compressive yield stress, i.e. it is positive in compression, as are $P_{1}$ and $P_{2}$, whilst $\ddot{\varepsilon}$ and $\ddot{\varepsilon}$ will typically have negative numerical values.

Earlier papers [2,3], by ignoring the uniform velocity field $v$, derived equations which only contained the first two expressions, e.g.

$$
P_{2}=\sigma_{y}-\rho \ddot{\varepsilon}\left(\frac{a^{2}}{8}-\frac{h^{2}}{6}\right) .
$$

A useful outcome of this result is that if $h=a \sqrt{3} / 2$, the inertia term is cancelled. Since, in a normal Hopkinson bar experiment, $\ddot{\varepsilon}$ is only significant early on in the test, where the specimen dimensions have not changed significantly, this is often used as a useful guide for specimen design. However, one must also confirm that, for the specimen design chosen, the other inertia terms in Eq. (2) are not significant compared to $\sigma_{y}$.

\subsection{Stress based analysis}

Forrestal produced equations which explicitly consider the radial dependence of the stress in the specimen. Assuming again that the material is incompressible, and using an elastic analysis, he produced the following equation for the hydrostatic pressure, $p$, in the specimen due to radial inertia:

$$
p(r)=\frac{\rho\left(a^{2}-r^{2}\right)}{4} \ddot{\varepsilon}_{z}
$$

here, $r$ is the radial position measured from the axis of the specimen and $\varepsilon_{z}$ the longitudinal strain. The obvious implication of this equation is that the hydrostatic pressure is largest at the centre of the specimen, as might be expected, and that the use of annular specimens might reduce the effect of inertia when performing measurements on weak or dense materials. This has been confirmed by experimental studies [6].

\subsection{Comparison of approaches}

The total additional stress predicted by the stress analysis may be calculated by integrating over the specimen

\footnotetext{
${ }^{1}$ A mistake in Gorham's Eq. (9a) has been corrected here, the original reads $P_{1}=\sigma_{y}+\rho \ddot{\varepsilon} \ldots$
}

surface, to give

$$
P_{\text {additional }}=\frac{1}{\pi a^{2}} \int_{0}^{a} \frac{\rho\left(a^{2}-r^{2}\right)}{4} \ddot{\varepsilon} 2 \pi r d r=\frac{\rho \ddot{\varepsilon} a^{2}}{8},
$$

such that, in the terminology of the energy based approach

$$
P_{1}=P_{2}=\sigma_{y}-\frac{\rho \ddot{\varepsilon} a^{2}}{8} .
$$

Because the analysis considers only a thin slice, $d z$, in the axial direction of the specimen, and because it assumes that the stress is constant in $z$, it does not give any difference between the stresses at the two bar-specimen interfaces, nor does it take into account the effects of the specimen height.

We will now modify the stress-based analysis, and show that it gives results consistent with the energy based approach. Firstly, it will be assumed that the material is behaving plastically according to a Mises yield criterion, and that the uniaxial yield stress is $\sigma_{y}$. It will also be assumed that $\sigma_{r}=\sigma_{\theta}$ and $\varepsilon_{r}=\varepsilon_{\theta}$. These assumptions are proved in Forrestal's elastic analysis, but will instead be assumed in this plastic treatment. We define the true strains in the axial and radial directions as $\varepsilon_{z}=\ln \left[h / h_{0}\right]$ and $\varepsilon_{r}=$ $\ln \left[r / r_{0}\right]$, and assume that the specimen is incompressible, so that $\varepsilon_{r}=-\varepsilon_{z} / 2$. The relationships between velocity and acceleration and true strain rate are given by

$$
u_{z}=-u=h \dot{\varepsilon}_{z} ; \dot{u}_{z}=-\dot{u}=h\left[\ddot{\varepsilon}_{z}+\dot{\varepsilon}_{z}^{2}\right]
$$

and

$$
u_{r}=r \dot{\varepsilon}_{r} ; \quad \ddot{u}_{r}=r\left[\ddot{\varepsilon}_{r}+\dot{\varepsilon}_{r}^{2}\right]=-\left[\frac{\ddot{\varepsilon}_{z}}{2}-\frac{\dot{\varepsilon}_{z}^{2}}{4}\right] .
$$

The calculations will proceed assuming that it is possible to treat the radial and axial motions separately, and a brief discussion of this assumption will be given later on.

Firstly, we write the equilibrium equation in the radial direction

$$
\frac{d \sigma_{r}}{d r}+\frac{\sigma_{r}-\sigma_{\theta}}{r}=-\rho \frac{d^{2} u_{r}}{d t^{2}},
$$

and, given that $\sigma_{r}=\sigma_{\theta}$ we note that

$$
\frac{d \sigma_{r}}{d r}=-\rho r\left[\ddot{\varepsilon}_{r}+\dot{\varepsilon}_{r}^{2}\right]
$$

given that $\sigma_{r}=0$ at $r=a$,

$$
\sigma_{r}=-\frac{\rho}{2}\left[\ddot{\varepsilon}_{r}+\dot{\varepsilon}_{r}^{2}\right]\left(a^{2}-r^{2}\right)=\frac{\rho}{2}\left[\frac{\ddot{\varepsilon}_{z}}{2}-\frac{\dot{\varepsilon}_{z}^{2}}{4}\right]\left(a^{2}-r^{2}\right) .
$$

Assuming a Mises yield criterion, and noting that whilst the $\sigma_{r}$ above is negative in compression the convention in this work is for compression to be positive, we can write that $\sigma_{z}+\sigma_{r}=\sigma_{y}$, such that

$$
\sigma_{z}=\sigma_{y}-\sigma_{r}=\sigma_{y}-\frac{\rho}{2}\left[\frac{\ddot{\varepsilon}_{z}}{2}-\frac{\dot{\varepsilon}_{z}^{2}}{4}\right]\left(a^{2}-r^{2}\right) .
$$

Compared to the analysis of Forrestal, this equation contains an extra term in $\dot{\varepsilon}_{z}^{2}$; this term is essentially due to 
the change in axial velocity required to deliver a constant true strain, and so appears in the relationship between axial acceleration and true strain rate.

This stress can now be integrated over the specimen surface to give

$$
\begin{aligned}
P_{1}^{\prime}=P_{2}^{\prime} & =\sigma_{y}-\frac{\rho}{8} a^{2}\left[\ddot{\varepsilon}_{z}-\frac{\dot{\varepsilon}_{z}^{2}}{2}\right] \\
& =\sigma_{y}-\frac{\rho}{8} a^{2} \ddot{\varepsilon}_{z}+\frac{\rho}{16} a^{2} \dot{\varepsilon}_{z}^{2},
\end{aligned}
$$

which agrees with the terms in $a^{2}$ from the energy based analysis.

In order to introduce axial variation, we note that

$$
\begin{aligned}
& \left(P_{1}-P_{2}\right) \pi a^{2}=m\left(\frac{\ddot{u}}{2}+\dot{v}\right) \\
& \rightarrow\left(P_{1}-P_{2}\right)=\rho h\left(\frac{-h\left[\ddot{\varepsilon}_{z}+\dot{\varepsilon}_{z}^{2}\right]}{2}+\dot{v}\right),
\end{aligned}
$$

and more generally that

$$
\begin{aligned}
& \left(P(z)-P_{2}\right) \pi a^{2}=m\left(\frac{\dot{u} z}{2 h}+\dot{v}\right) \\
& \rightarrow\left(P(z)-P_{2}\right)=\rho h\left(\frac{-\left[\ddot{\varepsilon}_{z}+\dot{\varepsilon}_{z}^{2}\right] z}{2 h}+\dot{v}\right),
\end{aligned}
$$

where $P(z)$ is introduced as the mean axial pressure at any axial position in the specimen.

Overall, then, we can say that

$$
\begin{aligned}
P_{2}= & \sigma_{y}-\frac{\rho}{8}+\frac{\rho}{16} \dot{\varepsilon}_{z}^{2} a^{2}+\text { constant } \\
P_{1}= & \sigma_{y}-\frac{\rho}{8} a^{2} \ddot{\varepsilon}_{z}+\frac{\rho}{16} a^{2} \dot{\varepsilon}_{z}^{2}+\text { constant } \\
& -\frac{\rho h^{2} \ddot{\varepsilon}_{z}}{2}-\frac{\rho h^{2} \dot{\varepsilon}_{z}^{2}}{2}+\rho h \dot{v} .
\end{aligned}
$$

These equations are therefore entirely consistent with the results from the energy-based analysis except that they give no indication of how to derive the constant, which we can see from the other analysis is in fact

$$
\frac{\rho h^{2} \ddot{\varepsilon}_{z}}{6}+\frac{\rho h^{2} \dot{\varepsilon}_{z}^{2}}{6}-\frac{\rho h \dot{v}}{2} .
$$

\section{Inertia in tension specimens}

The description of inertia in tension specimens will be split into three parts: use of the compressive equations with the directions reversed; addition of lumped masses; consideration of the shape of the specimen to give lower and upper bounds.

A typical tensile specimen design is a flat or cylindrical dogbone with a gauge section, shoulders and endcaps, Fig. 2(a). The simplest assumption is that the shoulders and endcaps do not exist, and that only the gauge length of the specimen is required, Fig. 2(b). This would be the case, for example, if the forces were measured at
Figure 2(a). Schematic diagram of a typical tensile test specimen.

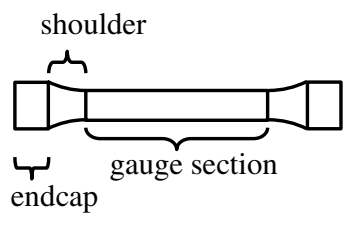

Figure 2(b). Specimen considered with no endcaps.

$$
\text { gauge section }
$$

Figure 2(c). Specimen approximated with non-deforming endcaps.
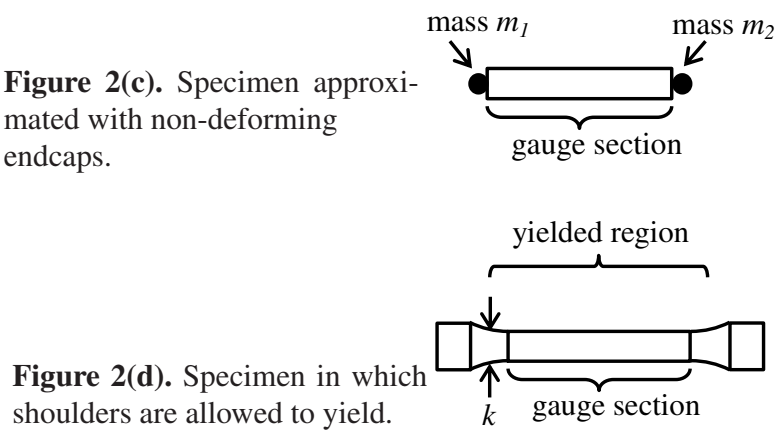

the end of the gauge section. The second assumption is equivalent to saying that the shoulders and endcaps do not deform, which might be a good approximation to the real behaviour, and instead just act as point masses on the end of the gauge length, Fig. 2(c). More realistically, some undefined portion of the shoulders deforms significantly, Fig. 2(d). In a perfect specimen of an elastic perfectly plastic material, plastic deformation would be confined to the gauge length, but because the radial inertia increases the axial stress required to yield the gauge length, plastic deformation would in fact enter the shoulders in a real tensile scimen deformed at high rates of strain.

\subsection{Reverse compression}

A detailed analysis is not presented here, but following the arguments of Samanta and Gorham shows that the mean end stresses in a cylindrical specimen loaded in tension are the reverse of those found in compression

$$
P_{1}=\sigma_{y}+\rho \ddot{\varepsilon}\left(\frac{a^{2}}{8}+\frac{h^{2}}{3}\right)-\rho \dot{\varepsilon}^{2}\left(\frac{a^{2}}{16}+\frac{h^{2}}{3}\right)+\frac{\rho h \dot{v}}{2}
$$

$$
P_{2}=\sigma_{y}+\rho \ddot{\varepsilon}\left(\frac{a^{2}}{8}-\frac{h^{2}}{6}\right)-\rho \dot{\varepsilon}^{2}\left(\frac{a^{2}}{16}+\frac{h^{2}}{6}\right)-\frac{\rho h \dot{v}}{2},
$$

where the directions of $P_{1}, P_{2}$ and $v$ are reversed and $\sigma_{y}$ is positive in tension. We note now that that $\dot{\varepsilon}$ and $\ddot{\varepsilon}$ will typically have positive numerical values.

\section{2. "Lumped Mass"}

If the endcaps do not deform, they may simply be considered as masses on the end of the cylindrical specimen. They must be accelerated, so work must be done to provide their kinetic energy; however, there is 

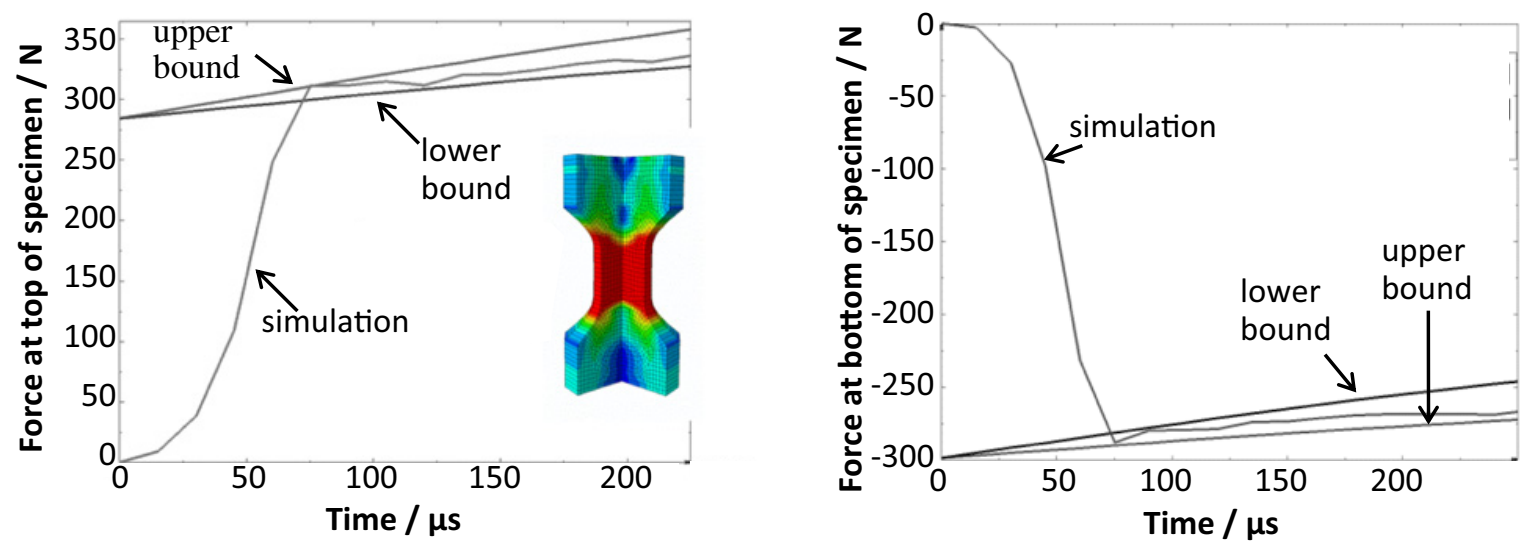

Figure 3. Comparison of specimen end forces taken from an FE simulation with upper and lower bound solutions. The specimen is shown.

no dormation energy. The two modifications made to the analysis of Samanta or Gorham are to state that

$\left(P_{1}-P_{2}\right) \pi b^{2}=\rho \pi a^{2} h\left(\frac{\dot{u}}{2}+\dot{v}\right)+m_{1}(\dot{u}+\dot{v})+m_{2} \dot{v}$

where $b$ is the radius of the endcaps, and $m_{1}, m_{2}$ their masses (usually $m_{1}=m_{2}$ ), and also to express the kinetic energy as

$$
\begin{aligned}
\dot{K} & =\dot{K}_{m 1}+\dot{K}_{m 2}+\dot{K}_{\text {cylinder }} \\
& =m_{1}(u+v)(\dot{u}+\dot{v})+m_{2} v \dot{v}+\dot{K}_{\text {cylinder }}
\end{aligned}
$$

where $\dot{K}_{\text {cylinder }}$ is the change in kinetic energy of the gauge section as calculated by Samanta and Gorham. It is noted that $P_{1}$ and $P_{2}$ are now defined at the end of the endcaps. The result of the analysis is

$$
\begin{aligned}
P_{1}= & \frac{a^{2}}{b^{2}} \sigma_{y}+\frac{m_{1}}{\pi b^{2}}(\dot{u}+\dot{v}) \\
& +\rho \frac{a^{2}}{b^{2}}\left[\ddot{\varepsilon}\left(\frac{a^{2}}{8}+\frac{h^{2}}{3}\right)-\dot{\varepsilon}^{2}\left(\frac{a^{2}}{16}+\frac{h^{2}}{3}\right)+\frac{\dot{v} h}{2}\right]
\end{aligned}
$$

$$
\begin{aligned}
P_{2}= & \frac{a^{2}}{b^{2}} \sigma_{y}-\frac{m_{2}}{\pi b^{2}} \dot{v} \\
& +\rho \frac{a^{2}}{b^{2}}\left[\ddot{\varepsilon}\left(\frac{a^{2}}{8}-\frac{h^{2}}{6}\right)-\dot{\varepsilon}^{2}\left(\frac{a^{2}}{16}+\frac{h^{2}}{6}\right)-\frac{\dot{v} h}{2}\right]
\end{aligned}
$$

so that the force on each end of the specimen is further modified by the mass of the endcap on that end of the specimen.

\subsection{Deformable shoulders}

In an ideal tensile specimen, the axial stress at the point where the gauge length meets the shoulders would be $\sigma_{y}$ (and all other stress components would be zero). In fact, this is not the case, and the axial stress is $\sigma_{y}+\sigma_{i}$ (where $\sigma_{i}$ is the inertial stress). Because the shoulders deform less than the specimen, it is likely that the inertial stress in the shoulders is less than $\sigma_{i}$, and therefore there is potential for the shoulders themselves to yield if the reduction in $\sigma_{i}$ is not sufficiently countered by an increase in radius. This leads to an increase in the work done to deform the material, and therefore an increase in the measured pressures at the ends of the bars.

The actual situation is complex, but may be simplified by thinking of upper and lower bounds. The lower bound on the additional stress is provided in the previous section (Eqs. (22) and (23)), because no additional deformation energy is required to be provided by $P_{1}$ and $P_{2}$. The upper bound will be calculated by assuming that the specimen yields to a new section whose radius is $k$, such that $\pi k^{2} \sigma_{y}=\pi a^{2}\left(\sigma_{y}+\sigma_{i}\right)$, where $\sigma_{i}$ is the axial stress owing to inertia in the gauge length. An overestimate of the additional work required is provided by $\dot{W}=\pi k^{2} u \sigma_{y}=$ $\pi a^{2} u\left(\sigma_{y}+\sigma_{i}\right)$; this is an overestimate because it assumes that the radius of the whole deforming region is $k$.

In these calculations, the assumption is made that we may use $u$ as the representative velocity for the work calculations. Until now, $u$ has been the velocity of the gauge section, now it is the velocity of the interface between yielded and yielded material in the shoulder. In practice, we may reconcile this by stating that $u$ is the relative velocity of the ends of the Hopkinson bars, which, during plastic deformation of the gauge section, will indeed be a good representation of the velocity of this interface (whether or not it coincides with the end of the gauge section). In any case, as the gauge section now moves more slowly than the shoulders, the real value of $\sigma_{i}$ will be less than we assume in this calculation. We will continue to use the equations described above for the relationship between $u, \dot{\varepsilon}_{z}^{2}$ and $\ddot{\varepsilon}$, even though we know that they are no longer precisely correct for the strains in the gauge length. It is possible to adopt an iterative approach to correct this, but this is likely to be too much effort in practice, and the current equations should be suitable as a design guideline. Despite these issues, the approximation is used because it gives a neat result which 
will be particularly suitable as a design guideline: the contribution of the cylinder inertia to the measured stress simply doubles:

$$
\sigma=\sigma_{y}+2 \sigma_{i}
$$

so that

$$
\begin{aligned}
P_{1}= & \frac{a^{2}}{b^{2}} \sigma_{y}+\frac{m_{1}}{\pi b^{2}}(\dot{u}+\dot{v}) \\
& +2 \rho \frac{a^{2}}{b^{2}}\left[\ddot{\varepsilon}\left(\frac{a^{2}}{8}+\frac{h^{2}}{3}\right)-\dot{\varepsilon}^{2}\left(\frac{a^{2}}{16}-\frac{h^{2}}{3}\right)-\frac{\dot{v} h}{2}\right]
\end{aligned}
$$$$
P_{2}=\frac{a^{2}}{b^{2}} \sigma_{y}-\frac{m_{2}}{\pi b^{2}} \dot{v}
$$$$
+2 \rho \frac{a^{2}}{b^{2}}\left[\ddot{\varepsilon}\left(\frac{a^{2}}{8}-\frac{h^{2}}{6}\right)-\dot{\varepsilon}^{2}\left(\frac{a^{2}}{16}+\frac{h^{2}}{6}\right)-\frac{\dot{v} h}{2}\right] .
$$

A number of Finite Element simulations have been performed with a range of different specimen geometries. The results from one of these simulations are compared to the upper and lower bound solutions in Fig. 3.

\section{Conclusions}

Equations have been derived for the effects of specimen inertia on the observed mechanical response of dog-bone specimens under dynamic tensile loading. In particular, by making assumptions about the yielding behaviour of the specimen outside the gauge length, it is possible to calculate quantitative lower and upper bounds on the extra stress observed owing to this inertia. The equations produced can be used as design guidelines for high strain rate tensile specimens.

\section{References}

[1] H. Kolsky, Proc. Phys. Soc. B62 (1949)

[2] E.D.H. Davies and S.C. Hunter, J. Mech. Phys. Solids 11 (1963)

[3] S.K. Samanta, J. Mech. Phys. Solids 19 (1971)

[4] D.A. Gorham, J. Phys. D: Appl. Phys. 22 (1989)

[5] M.J. Forrestal, T.W. Wright, W. Chen, International Journal of Impact Engineering 34 (2007)

[6] B. Song, Y. Ge, W.W. Chen T. Weerasooriya, Experimental Mechanics 47 (2007) 